\title{
SHAPES OF MAGELLANIC CLOUD STAR CLUSTERS
}

\author{
E. KONTIZAS, M. KONTIZAS \\ Laboratory for Astronomy and Solar Physics \\ NASA Goddard Space Flight Centre \\ Greenbelt, MD 2077 \\ USA \\ G. SEDMAK, R. SMAREGLIA \\ Osservatorio Astronomico di Trieste \\ Via Tiepolo 11 \\ Trieste 34131 \\ Italy
}

\begin{abstract}
The ellipticities of the most populous MC star clusters (87) have been derived for studying a) ellipticity variations within individual clusters, b) variations of ellipticity distributions for various galaxies, and c) variations of ellipticity of star clusters relative to their dynamical parameters.

From the studied clusters it is found that radial ellipticity variations within individual clusters seem to be a common phenomenon for the MC clusters where the inner parts are more elliptical than the outer. The SMC clusters are more elliptical than LMC clusters where ellipticities are measured at $r_{h}$.

The dynamical masses of the clusters seem to correlate with ellipticities where the most massive are the most elliptical.
\end{abstract}

\section{Observations and reductions}

The morphology of stellar systems (in particular their geometry), can provide important insights into their dynamics. The non-spherical appearance of globular clusters was first noted by Pease and Shapley (1917).

To study the shape of LMC and SMC globular clusters, U, J and R plates taken with the $1.2 \mathrm{~m}$ U.K. Schmidt telescope were digitized using a P.D.S. 1010A at the Trieste Observatory. The images could be scrolled and zoomed before filtering. The filter is of ranked type (Pasian 1988) and its parameters (order of rank) are defined interactively for the best compromise between the smoothness and the distortion of filtered images. We estimate the error arising from the selection of the best fit ellipse to be 0.03 . Simulations in our data have shown that the error due to background effect can be as large as 0.04 for BFLAT smaller than 0.1 (Kontizas et al. 1989). Geisler and Hodge (1980) found that errors due to resolution effect can range up to 0.04 for the spherical clusters but become much smaller for the elliptical. From the above estimates of various sources of errors, variability within a cluster or different clusters is considered significant if it exceeds 0.06. Derived ellipticities for LMC/SMC clusters have been published previously (Kontizas et al. 1989, 1990). 


\section{Conclusions}

The results of this investigation can be summarized as follows.

There is clear evidence for an intrinsic variation of cluster ellipticity and consequently, when referring to an ellipticity, we must define the distance from the cluster centre where it is measured. Our results were compared to previously given values of ellipticities, where the corresponding distance from the cluster centre was given, and they have been found to agree very well within our errors.

The ellipticities were found to increase towards the central regions of the clusters. The Kolmogorov-Smimov test showed that ellipticities of the inner and outer regions come from different populations with a confidence level of $99 \%$.

The ages of the clusters from the point of view of stellar evolution, and the ellipticities of the clusters at $r_{h}$ do not seem to correlate; although, when ellipticities are measured at the outer regions there is an indication that the young ones are somehow flatter.

The SMC clusters are more elliptical than those in the LMC, and, in turn, LMC clusters are more elliptical than those of our Galaxy (Kontizas et al. 1990).

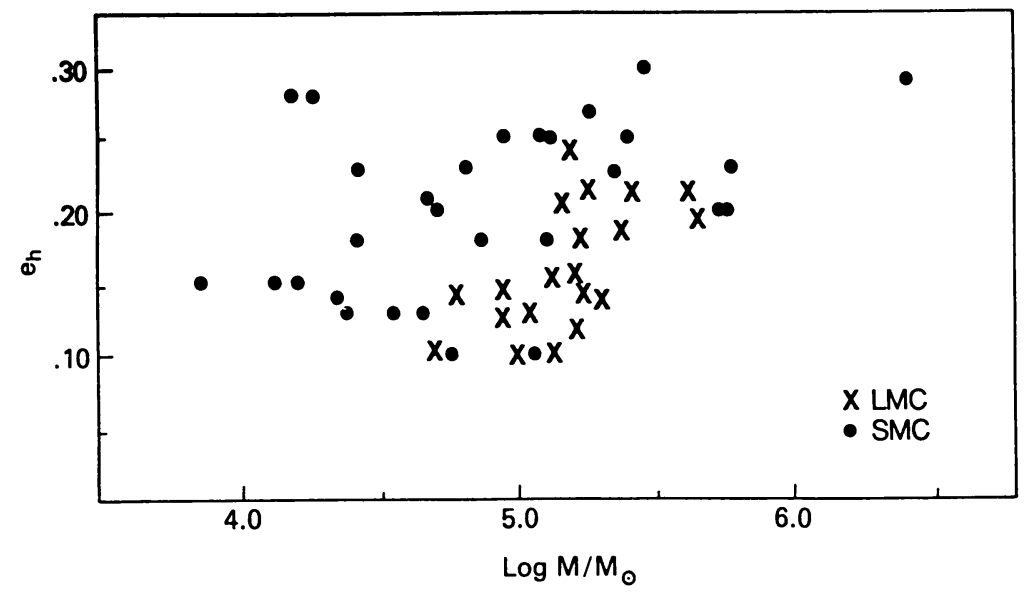

Figure 1. Ellipticities at $r_{h}(v s)$ the dynamical masses

Finally, Fig 1 shows that the most massive LMC and SMC clusters are the most elliptical.

\section{References}

Geisler, D. and Hodge, P.W. (1980), Astrophys. J. 242, 66.

Kontizas, E., Kontizas, M., Sedmac, G., Smareglia, R. (1989), Astron. J. 98, 590.

Kontizas, E., Kontizas, M., Sedmac, G., Smareglia, R., Dapergolas, A. (1990), Astron. J. (in press).

Pasian, F. (1988), Mem. Soc. Astron. Ital. 59, 337.

Pease, F.G. and Shapley, H. (1917), Contr. Mt. Wilson Obs. 129. 